\title{
On the Probability in General Physics from the Perspective of the Energy Structure
}

\author{
Saeed Shahsavari \\ Department of Mechanical Engineering, Isfahan University of Technology \\ Isfahan, Iran. \\ Email: s.shahsavari [AT] me.iut.ac.ir
}

\begin{abstract}
What are the all final possible states that a physical system can reach when some energy is applied to the system? This question can be known as one of the physical questions that relate to the probability in physics. The second law of thermodynamics is known as the base of the probability concept that is raised in modern physics, while this concept is extracted in physical theories by different meanings. As a new approach to investigate the probability, at first, the energy space concept is extracted and then by applying the effects of the second law of thermodynamics on it, the energy structure is presented. The energy structure of the system is a new perspective to investigate the probability, and by using it, the relation between all possible accessible states, when some energy is applied to the system, can be determined.
\end{abstract}

Keywords- Energy Space; Energy Structure; Second Law of Thermodynamics; Probability; Feasible processes

\section{INTRODUCTION}

The concept of probability is raised in modern physics theories as a new important approach to nature, while this concept is not used in different theories by the same meaning. The non-equality of the second law of thermodynamics is known as the base of the probability concept in science. Quantum mechanics and classical statistical physics are two important theories that probability is raised by them. Supernaturally, quantum mechanics establishes irreducible probabilistic claims about some physical systems. As radioactive decay, atomic transitions, and the like. More intentionally, classical statistical physics establishes extensive use of probability concepts to investigate the classical microstates [1-4].

Stochastic dynamics probability is one of the meanings of probability in physics. Based on the stochastic dynamics, the probability concept means the possible historical states of the world, while one of them is actual [5-8]. The concept of probability is used in statistical mechanics to determine the distributions of energy levels. Statistical mechanics use this meaning of probability to investigate the macro states by investigating the micro states [9-11]. Quantum mechanics uses the concept of probability to determine the state space of the quantum systems [12-16]. When a particular state is determined, the Born rule can be used to translate the state to the probabilistic state, and also the dynamics of a probability distribution can be determined [17-19]. In this paper, we extract a new approach to the effects of the probability of physical systems and all possible final states when some energy is applied to the system, is extracted also.

\section{ENERGY SPACE AND ENERGY STRUCTURE}

Each physical system has a set of energy components that can be activated in applying energy. The set of these components can be known as the energy space of the system that when a physical process is performed, some of these components are activated. From the perspective of the energy space, it can result that the different states in quantum state space, and also different distributions in statistical physics, have the same activated energy components in a particular process. This concept can introduce a new approach to investigate probability effects in physical systems.

When a physical process is performed, some of the components of the energy space are activated. Some of the activated components will be dependent on the remaining, as an dependent component. By determining the general dependence between independent and dependent components, the energy structure will be extracted. To apply the effects of the irreversibility and probability on the energy structure of the system, the dependent components must be considered as functions of the amount or the rate of independent components [20-22]. Therefore:

$U_{E-C}=\left(u_{1}+u_{2}+\cdots+u_{m}\right)+\left[g_{1}+\cdots+g_{k}\right]+\left[h_{1}+\cdots+h_{n}\right]$

Where:

$g_{j}=g_{j}\left(u_{1}, u_{2}, \ldots, u_{m}\right)$ 
$h_{p}=h_{p}\left(\dot{u}_{1}, \ldots, \dot{u}_{m}\right)$

That $U_{E-C}$ is the energy changed in the process, $u_{i}$ are independent components, $g_{j}$ and $h_{p}$ are dependent components of energy. The components $h_{p}$ do not act in quasi-static conditions.

\section{PROBABILITY FROM THE PERSPECTIVE OF THE ENERGY STRUCTURE}

Equation (1) introduces a new approach to the probability in physical processes. When some energy is applied to the system, different possible states is extracted by diffusion this amount of energy into the activated components so that equation (1) be established. If $\delta u_{i-1}$ and $\delta u_{i-2}$ show two possible changes of an independent component $u_{i}$ when some energy is applied to the system, as shown in figure 1, Therefore all possible paths must establish equation (4):

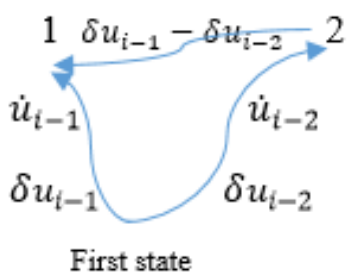

Figure 1. Possible paths, when some energy is applied to the system

$\delta u_{i-1}+\sum_{j=1}^{k}\left(\frac{\partial g_{j}}{\partial u_{i}} \delta u_{i-1}\right)+\sum_{p=1}^{n} h_{p}\left(\dot{u}_{i-1}\right)=\delta u_{i-2}+\sum_{j=1}^{k}\left(\frac{\partial g_{j}}{\partial u_{i}} \delta u_{i-2}\right)+\sum_{p=1}^{n} h_{p}\left(\dot{u}_{i-2}\right)$

Or:

$\left[\frac{\left(1+\sum_{j=1}^{k}\left(\frac{\partial g_{j}}{\partial u_{i}}\right)\right)}{\sum_{p=1}^{n}\left[h_{p}\left(\dot{u}_{i-1}\right)-h_{p}\left(\dot{u}_{i-2}\right)\right]}\right]\left(\delta u_{i-1}-\delta u_{i-2}\right)=1$

From the perspective of the kinetic theory of dissipated energy, it can be concluded that $h_{p}$ can be considered as a linear function [22-25]. Therefore, by using the variable change $\delta U_{i}=\delta u_{i-1}-\delta u_{i-2}$, equation (5) can be rewritten as follows:

$\left[\frac{\left(1+\sum_{j=1}^{k}\left(\frac{\partial g_{j}}{\partial u_{i}}\right)\right)}{\sum_{p=1}^{n}\left[h_{p}\left(\dot{U}_{i}\right)\right]}\right]\left(\delta U_{i}\right)=1$

Equation (6) depends on the condition of applying energy to the system. To establish the principle of the kinetic theory of dissipated energy [22-24], $\sum_{p=1}^{n}\left[h_{p}\left(\dot{U}_{i}\right)\right]$ must have opposite sign with $\dot{U}_{i}$. Therefore, relation (7) can be concluded as the relation between all final possible states:

$\left(1+\sum_{j=1}^{k}\left(\frac{\partial g_{j}}{\partial u_{i}}\right)\right)\left[\delta\left(U_{i}^{2}\right)\right] \geq 0$

Relation (7) is not depending on the condition of the applying energy and extracted the relation between all final possible states as a non-equality. This relation has the same base as the second law of thermodynamics as the base of probability in physics [25-28].

\section{CONCLUSIONS}

The concept of probability is raised in science by modern theories of physics. The base of probability in physics is the second law of thermodynamics. From the perspective of the energy structure, a new general approach to the probability can be extracted. Relation (7) have the probability base, and also the same base as the second law of thermodynamics. This relation is not depending on the condition of applying energy, and also extracts all possible final states when some energy is applied to the system.

\section{REFERENCES}

[1] Birkhoff, G. D. "Probability and physical systems." Bulletin of the American Mathematical Society 38.6 (1932): 361 379.

[2] Galavotti, Maria Carla. "What Interpretation for Probability in Physics?." Chance in Physics. Springer, Berlin, Heidelberg, 2001. 265-269. 
[3] Kac, Mark, ed. Probability and related topics in physical sciences. Vol. 1. American Mathematical Soc., 1959.

[4] Ben-Menahem, Yemima, and Meir Hemmo, eds. Probability in physics. Springer Science \& Business Media, 2012.

[5] Chandrasekhar, Subrahmanyan. "Stochastic problems in physics and astronomy." Reviews of modern physics 15.1 (1943): 1 .

[6] Lemons, Don S., and Paul Langevin. An introduction to stochastic processes in physics. JHU Press, 2002.

[7] Li, Jie, et al. "Advances of the probability density evolution method for nonlinear stochastic systems." Probabilistic Engineering Mechanics 28 (2012): 132-142.

[8] Schrödinger, Erwin. "Discussion of probability relations between separated systems." Mathematical Proceedings of the Cambridge Philosophical Society. Vol. 31. No. 4. Cambridge University Press, 1935.

[9] Guttmann, Yair M. The concept of probability in statistical physics. Cambridge University Press, 1999.

[10] Chakrabarti, C. G., and Indranil Chakrabarty. "Boltzmann entropy: probability and information." arXiv preprint arXiv:0705.2850 (2007).

[11] Obregón, Octavio, and Alejandro Gil-Villegas. "Generalized information entropies depending only on the probability distribution." Physical Review E 88.6 (2013): 062146.

[12] Caves, Carlton M., Christopher A. Fuchs, and Rüdiger Schack. "Subjective probability and quantum certainty." Studies in History and Philosophy of Science Part B: Studies in History and Philosophy of Modern Physics 38.2 (2007): 255-274.

[13] Rédei, Miklós, and Stephen Jeffrey Summers. "Quantum probability theory." Studies in History and Philosophy of Science Part B: Studies in History and Philosophy of Modern Physics 38.2 (2007): 390-417.

[14] Paty, Michel. "Physical quantum states and the meaning of probability." (2001).

[15] Robinett, R. W. "Quantum and classical probability distributions for position and momentum." American Journal of Physics 63.9 (1995): 823-832.

[16] Saunders, Simon. "What is probability?." Quo vadis quantum mechanics?. Springer, Berlin, Heidelberg, 2005. 209238.

[17] Jauch, Josef M. "The quantum probability calculus." Logic and probability in stochaal mechanics. Springer, Dordrecht, 1976. 123-146.

[18] Man'ko, Margarita A., and Vladimir I. Man'ko. "Probability description and entropy of classical and quantum systems." Foundations of Physics 41.3 (2011): 330-344.

[19] Domotor, Zoltan. "The probability structure of quantum-mechanical systems." Logic and Probability in Quantum Mechanics. Springer, Dordrecht, 1976. 147-177.

[20] Lucia, Umberto. "Probability, ergodicity, irreversibility and dynamical systems." Proceedings of the Royal Society A: Mathematical, Physical and Engineering Sciences 464.2093 (2008): 1089-1104.

[21] Chen, Jian-Bing, and Jie Li. "A note on the principle of preservation of probability and probability density evolution equation." Probabilistic Engineering Mechanics 24.1 (2009): 51-59.

[22] Jiang, Da-Quan, Donghua Jiang, and Min Qian. Mathematical theory of nonequilibrium steady states: on the frontier of probability and dynamical systems. No. 1833. Springer Science \& Business Media, 2004.

[23] Thomson, William. "9. The kinetic theory of the dissipation of energy." Proceedings of the Royal Society of Edinburgh 8 (1875): 325-334.

[24] Burbury, S. H. "LII. A theorem on the dissipation of energy." The London, Edinburgh, and Dublin Philosophical Magazine and Journal of Science 13.83 (1882): 417-419.

[25] Shahsavari, Saeed, Mehran Moradi, "A Study of the Entropy Production in Physical Processes from a New Perspective of the Energy Structure." Asian Journal of Applied Sciences (ISSN: 2321-0893) 8.6 (2020).

[26] Shahsavari, Saeed, Mehran Moradi, and Morteza Esmaeilpour. "On the Irreversibility in Mechanical Systems Using a New Macroscopic Energy Structure Modeling." Asian Journal of Applied Sciences (ISSN: 2321-0893) 8.6 (2020). 
[27] Shahsavari, Saeed, Mehran Moradi, and Morteza Esmaeilpour. "On the Available Work Limits at Constant Heat and Entropy Production." Asian Journal of Applied Sciences (ISSN: 2321-0893) 8.6 (2020).

[28] Shahsavari, Saeed, Mehran Moradi, " An Applied Component Modeling to the Irreversibility from a New Configurationally Perspective of the Statistical Physics." Asian Journal of Fuzzy and Applied Mathematics (ISSN: 2321 564X) 8.3 (2020). 